\title{
A Nonlinear Consensus Protocol of Multiagent Systems Considering Measuring Errors
}

\author{
Xiaochu Wang, Kaichun Zhao, Zheng You, and Lungui Zheng \\ State Key Laboratory of Precision Measurement Technology and Instruments, Tsinghua University, Beijing 100084, China \\ Correspondence should be addressed to Kaichun Zhao; kaichunz@mail.tsinghua.edu.cn \\ and Zheng You; yz-dpi@mail.tsinghua.edu.cn
}

Received 29 May 2013; Revised 20 August 2013; Accepted 30 August 2013

Academic Editor: Kwok-Wo Wong

Copyright (C) 2013 Xiaochu Wang et al. This is an open access article distributed under the Creative Commons Attribution License, which permits unrestricted use, distribution, and reproduction in any medium, provided the original work is properly cited.

In order to avoid a potential waste of energy during consensus controls in the case where there exist measurement uncertainties, a nonlinear protocol is proposed for multiagent systems under a fixed connected undirected communication topology and extended to both the cases with full and partial access a reference. Distributed estimators are utilized to help all agents agree on the understandings of the reference, even though there may be some agents which cannot access to the reference directly. An additional condition is also considered, where self-known configuration offsets are desired. Theoretical analyses of stability are given. Finally, simulations are performed, and results show that the proposed protocols can lead agents to achieve loose consensus and work effectively with less energy cost to keep the formation, which have illustrated the theoretical results.

\section{Introduction}

One of the most attractive benefits of multiagent systems is that many fractional, inexpensive, and simple agents working together can achieve the same objective as a monolithic, expensive, and complicated agent. Since certain formation configurations are often indispensable for such systems, the coordination problem in the area of formation control has attracted compelling interest in recent years. One critical issue arising from the coordination problem is to develop distributed control laws based on local information that enable all agents to reach an agreement on certain quantities of interest, which is known as the consensus problem.

In the context of multiagent systems, consensus problems have been studied widely in recent years. Reference [1] provided a theoretical explanation for the coordination behavior of a group of autonomous agents, and results implied that proper control models based only on neighbor information could motivate systems to achieve consensus. Reference [2] provided an overview of information consensus in multivehicle cooperative control, with several consensus control laws reviewed by describing some specific coordination applications. More challenging results were obtained by extending the simple first-order dynamics to second-order ones to better solve practical problems with some actual conditions being considered, such as actuator saturations and lacks of relative velocity measurements (see, e.g., [3]). And, more applications of consensus can be also found in varieties of research paper, such as flocking, attitude alignment, swarm moving, and containment control (see, e.g., [4-7]).

Most results of the aforementioned research are obtained in case where dynamics are linear. And in case where the dynamic agents are physical models, the design and analysis of nonlinear consensus protocols have also been studied, with input constraints of such systems being taken into account for more adaptive designs. Reference [8] introduced both linear and nonlinear protocols based on consensus for a group of dynamic agents with undirected information flow, and it implied from the definition of nonlinear functions that linear protocols are actually the special cases of the nonlinear ones. Reference [9] studied the performance of a class of special nonlinear protocols, which could be designed using any mean of order of the agents' states instead of the average mean to form the feedback term, to achieve consensus. Many other research papers have also been done to explore nonlinear protocols, such as $[10,11]$, where nonlinear protocols were 
utilized to achieve faster convergence or apply with secondorder dynamic agents, respectively.

However, sometimes, energy means a lot for some particular applications, such as certain applications for space, for example, formation keeping and attitude alignment. It is worthwhile to note that, although the aforementioned protocols are able to deal with the measurement uncertainties, they may not perform well in saving energy (which will be shown through comparisons in the later simulations). It can be known that, in the typical tight spacecraft formation keeping missions, the relative position measurements are of precision of centimeter number grade [12]. So, due to the existence of measurement uncertainties, it is unnecessary that the aforementioned protocols should act even when the measured position configuration errors are of precision of centimeter number grade. These overactive actions would cost energy continuously, but in the meanwhile, energy quantity influences the lifetimes of spacecraft for normal performances. Therefore, it is worthwhile to present a particular nonlinear protocol to address such uncertain problems for better saving of energy.

The outline of this paper is as follows. Some backgrounds on algebraic graph theory and linear protocol are given in Section 2. A nonlinear protocol without a reference is presented to achieve a loose consensus with its stability proof given together in Section 3.1. The protocol is extended to the case where all agents can communicate with a group references in Section 3.2. By introducing a set of sliding mode estimators to estimate a group reference for each agent, the protocol is easily extended to the case where only a portion of agents can communicate with a group reference in Section 3.3. Simulation results are presented in Section 4. Finally, concluding remarks are made in Section 5 .

\section{Backgrounds and Preliminaries}

The $m$-dimensional open-loop dynamics of $n$ agents with fixed undirected communication links can be given by

$$
\dot{\mathbf{r}}_{i}(t)=\mathbf{v}_{i}(t) \quad \dot{\mathbf{v}}_{i}(t)=\mathbf{u}_{i}(t), \quad i \in\{1, \ldots, n\} .
$$

A weighted undirected graph $\mathscr{G}=(\mathscr{V}, \mathscr{E})$ can be borrowed to model the interaction among these agents, where $\mathscr{V}=\{1, \ldots, n\}$ is the node set and $\mathscr{E} \subseteq \mathscr{V} \times \mathscr{V}$ is the edge set. If $(j, i) \in \mathscr{E}$, agents $i$ and $j$ can be called neighbors, which means that they can exchange information from each other. The weighted adjacency matrix $\mathscr{A}=\left[a_{i j}\right] \in R^{n \times n}$ associated with $\mathscr{G}$ is defined as $a_{i j}>0$ if $(j, i) \in \mathscr{E}$, while $a_{i j}=0$ otherwise. Since $(j, i) \in \mathscr{E}$ implies $(i, j) \in \mathscr{E}, a_{i j}=a_{j i}$ holds. The Laplacian matrix $\mathscr{L}=\left[\ell_{i j}\right] \in R^{n \times n}$ associated with $\mathscr{G}$ is defined as $\ell_{i i}=\sum_{j=1, j \neq i}^{n} a_{i j}$, while $\ell_{i j}=-a_{i j}$ for any $i \neq j$. It is clear that $\mathscr{A}$ is symmetric and $\mathscr{L}$ is symmetric positive semidefinite.
The linear consensus protocol can be given by (see, e.g., $[2,13])$

$$
\begin{aligned}
\mathbf{u}_{i}(t)= & -\sum_{j=1}^{n} a_{i j}\left(\mathbf{r}_{i}(t)-\mathbf{r}_{j}(t)\right) \\
& -\sum_{j=1}^{n} a_{i j} \gamma_{c}\left(\mathbf{v}_{i}(t)-\mathbf{v}_{j}(t)\right),
\end{aligned}
$$

where the gain $\gamma_{c}$ is a positive scalar. It follows from [13] that the protocol (2) can guarantee the consensus of the multiagent system (1) under any undirected connected interaction graph and the system is asymptotically stable with $\mathbf{r}_{i}(t) \rightarrow$ $\mathbf{r}_{j}(t)$ and $\mathbf{v}_{i}(t) \rightarrow 0$ asymptotically, as $t \rightarrow \infty$.

It is clear to note that protocol (2) would act even when the configuration errors are of the same precision as those of the measurements with costing energy unnecessarily. Assuming that measurement uncertainties are bounded, the mathematical forms can be written as follows:

$$
\begin{gathered}
\left\|\mathbf{r}_{i}(t)-\overline{\mathbf{r}}_{i}(t)\right\|_{\infty} \leq \varepsilon_{r} \\
\left\|\mathbf{v}_{i}(t)-\overline{\mathbf{v}}_{i}(t)\right\|_{\infty} \leq \varepsilon_{v},
\end{gathered}
$$

where $\overline{\mathbf{r}}_{i}(t)$ and $\overline{\mathbf{v}}_{i}(t)$ denote the true values, $\varepsilon_{r}$ and $\varepsilon_{v}$ denote the boundaries of errors, and $\|\cdot\|_{\infty}$ denotes the $\infty$-norm operator $\left(\|\mathbf{p}\|_{\infty}\right.$ equals the maximum absolute value of the components of vector $\mathbf{p}$ ).

\section{Consensus Analysis}

3.1. Nonlinear Protocol for Average. Intuitively thinking, when the configuration errors are of the same precision as those of the measurements, it is not convincing to control based on the measurements, and, instead, a conservative control strategy is considered. Introduce two nonlinear functions $\phi(z)$ and $\varphi(z)$, which are defined componentwise as:

$$
\begin{gathered}
\phi(z)= \begin{cases}z+\varepsilon_{r}, & z \in\left(-\infty,-\varepsilon_{r}\right], \\
0, & z \in\left[-\varepsilon_{r}, \varepsilon_{r}\right], \\
z-\varepsilon_{r}, & z \in\left[\varepsilon_{r},+\infty\right),\end{cases} \\
\varphi(z)= \begin{cases}z+\varepsilon_{v}, & z \in\left(-\infty,-\varepsilon_{v}\right], \\
0, & z \in\left[-\varepsilon_{v}, \varepsilon_{v}\right], \\
z-\varepsilon_{v}, & z \in\left[\varepsilon_{v},+\infty\right) .\end{cases}
\end{gathered}
$$

Then the nonlinear consensus protocol is given by

$$
\begin{aligned}
\mathbf{u}_{i}(t)= & -\sum_{j=1}^{n} a_{i j} \phi\left[\mathbf{r}_{i}(t)-\mathbf{r}_{j}(t)\right] \\
& -\sum_{j=1}^{n} a_{i j} \gamma_{c} \varphi\left[\mathbf{v}_{i}(t)-\mathbf{v}_{j}(t)\right], \quad \forall i \in\{1, \ldots, n\} .
\end{aligned}
$$

Before going on, a lemma and its proof are given as follows. 
Lemma 1. Suppose that $\varsigma \in R^{m}, \varphi \in R^{m}$, and $\mathbf{C}=\left[c_{i j}\right] \in R^{n \times n}$. If $\mathbf{C}$ is symmetric and $\phi(z)$ is an odd function, then it follows that

$$
\begin{array}{r}
\frac{1}{2} \sum_{i=1}^{n} \sum_{j=1}^{n} c_{i j}\left(\varsigma_{i}-\varsigma_{j}\right)^{T} \phi\left(\varphi_{i}-\varphi_{j}\right) \\
=\sum_{i=1}^{n} \sum_{j=1}^{n} c_{i j} \varsigma_{i}^{T} \phi\left(\varphi_{i}-\varphi_{j}\right) .
\end{array}
$$

Proof. Considered

$$
\begin{aligned}
& \frac{1}{2} \sum_{i=1}^{n} \sum_{j=1}^{n} c_{i j}\left(\varsigma_{i}-\varsigma_{j}\right)^{T} \phi\left(\varphi_{i}-\varphi_{j}\right) \\
& =\frac{1}{2} \sum_{i=1}^{n} \sum_{j=1}^{n} c_{i j} \varsigma_{i}^{T} \phi\left(\varphi_{i}-\varphi_{j}\right)-\frac{1}{2} \sum_{i=1}^{n} \sum_{j=1}^{n} c_{i j} \varsigma_{j}^{T} \phi\left(\varphi_{i}-\varphi_{j}\right) \\
& =\frac{1}{2} \sum_{i=1}^{n} \sum_{j=1}^{n} c_{i j} \varsigma_{i}^{T} \phi\left(\varphi_{i}-\varphi_{j}\right)+\frac{1}{2} \sum_{j=1}^{n} \sum_{i=1}^{n} c_{j i} \varsigma_{j}^{T} \phi\left(\varphi_{j}-\varphi_{i}\right) \\
& =\sum_{i=1}^{n} \sum_{j=1}^{n} c_{i j} \varsigma_{i}^{T} \phi\left(\varphi_{i}-\varphi_{j}\right),
\end{aligned}
$$

where, in order to get (7), we have used the facts that $c_{i j}=c_{j i}$ and $\phi\left(\varphi_{i}-\varphi_{j}\right)=-\phi\left(\varphi_{j}-\varphi_{i}\right)$, and we also have switched the dummy variables $i$ and $j$ in the summation function.

Theorem 2. Using protocol (5) for multiagent system (1), then if the communication topology graph of (1) is undirected and connected, loose consensus can be achieved so that $\left\|\mathbf{v}_{i}(t)-\mathbf{v}_{j}(t)\right\|_{\infty} \leq \varepsilon_{v}$ and $\left\|\mathbf{r}_{i}(t)-\mathbf{r}_{j}(t)\right\|_{\infty} \leq \rho$ as $t \rightarrow \infty$, for all $i, j$, where $\rho$ is a certain bounded scalar.

Proof. Denote $\psi(z)$ as the potential function of $\phi(z)$, which is also defined component-wise. One obtains that

$$
\psi(z)=\int_{0}^{z} \phi(s) d s .
$$

Introduce a Lyapunov function as

$$
V=\frac{1}{2} \sum_{i=1}^{n} \sum_{j=1}^{n} a_{i j} \mathbf{1}_{3}^{T} \psi\left(\mathbf{r}_{i}-\mathbf{r}_{j}\right)+\frac{1}{2} \sum_{i=1}^{n} \mathbf{v}_{i}^{T} \mathbf{v}_{i} .
$$

Then the time derivative of $V$ along the trajectories of (5) is given by

$$
\begin{aligned}
\dot{V}= & \frac{1}{2} \sum_{i=1}^{n} \sum_{j=1}^{n} a_{i j}\left(\mathbf{v}_{i}-\mathbf{v}_{j}\right)^{T} \phi\left(\mathbf{r}_{i}-\mathbf{r}_{j}\right) \\
& -\sum_{i=1}^{n} \mathbf{v}_{i}^{T} \sum_{j=1}^{n} a_{i j}\left[\phi\left(\mathbf{r}_{i}-\mathbf{r}_{j}\right)+\varphi\left(\mathbf{v}_{i}-\mathbf{v}_{j}\right)\right] \\
= & \sum_{i=1}^{n} \sum_{j=1}^{n} a_{i j} \mathbf{v}_{i}^{T} \phi\left(\mathbf{r}_{i}-\mathbf{r}_{j}\right) \\
& -\sum_{i=1}^{n} \sum_{j=1}^{n} a_{i j} \mathbf{v}_{i}^{T}\left[\phi\left(\mathbf{r}_{i}-\mathbf{r}_{j}\right)+\varphi\left(\mathbf{v}_{i}-\mathbf{v}_{j}\right)\right]
\end{aligned}
$$

$$
\begin{aligned}
& =-\sum_{i=1}^{n} \sum_{j=1}^{n} a_{i j} \mathbf{v}_{i}^{T} \varphi\left(\mathbf{v}_{i}-\mathbf{v}_{j}\right) \\
& =-\frac{1}{2} \sum_{i=1}^{n} \sum_{j=1}^{n} a_{i j}\left(\mathbf{v}_{i}-\mathbf{v}_{j}\right)^{T} \varphi\left(\mathbf{v}_{i}-\mathbf{v}_{j}\right)
\end{aligned}
$$

$\leq 0$,

where we have used derivative rules to obtain the first equality, have used Lemma 1 to obtain the second and fourth equality, and have used the fact that $\left(\mathbf{v}_{i}-\mathbf{v}_{j}\right)^{T} \varphi\left(\mathbf{v}_{i}-\mathbf{v}_{j}\right)$ is nonnegative to get the final inequality.

Let $S=\left\{\left(\mathbf{r}_{i}-\mathbf{r}_{j}, \mathbf{v}_{i}-\mathbf{v}_{j}\right) \mid \dot{V}=0\right\}$. Since $\dot{V} \leq 0$ along the trajectories of system, there exists $T>0$ such that $\dot{V} \equiv 0$ holds for all $t>T$. Note that $\dot{V} \equiv 0$ means $\varphi\left(\mathbf{v}_{i}-\mathbf{v}_{j}\right)=0$, for all $i, j$. So, when the undirected graph is connected, $\dot{V} \equiv 0$ implies $\left\|\mathbf{v}_{i}(t)-\mathbf{v}_{j}(t)\right\|_{\infty} \leq \varepsilon_{v}$, for all $i, j$. Therefore, it follows from (5) that

$$
\dot{\mathbf{v}}_{i}(t)=-\sum_{j=1}^{n} a_{i j} \phi\left[\mathbf{r}_{i}(t)-\mathbf{r}_{j}(t)\right],
$$

for all $t>T$.

It can be seen from (11) that agent $i$ is forced towards the weighted average position of its neighbors and it is the same case with the other agents. This means that all agents tend to gather. Combining with $\left\|\mathbf{v}_{i}(t)-\mathbf{v}_{j}(t)\right\|_{\infty} \leq \varepsilon_{v}$, for all $i$, $j$, one can obtain the conclusion that $\dot{\mathbf{v}}_{i}(t)$ is bounded, for all $i$. It then follows from (11) that there must exists a bounded scalar $\rho>0$ such that $\left\|\mathbf{r}_{i}(t)-\mathbf{r}_{j}(t)\right\|_{\infty} \leq \rho$ holds for all $t>T$. This completes the proof.

3.2. Consensus with All Accesses to a Group Reference. For the sake of being referred conveniently, if there exists a group reference which is available to all agents, the group reference can be labeled as a virtual agent 0 , with $a_{i 0}>0$, for all $i \in\{1, \ldots, n\}$ defined additionally as the weighted communication links. And it is worthwhile to note that the reference is not treated as a member of the $n$-agent system in this work actually, although it is labeled as number 0 . Thus the loose consensus protocol for the real $n$-agent system is given by

$$
\begin{aligned}
\mathbf{u}_{i}(t)= & \dot{\mathbf{v}}_{0}(t)-\sum_{j=0}^{n} a_{i j} \phi\left[\mathbf{r}_{i}(t)-\mathbf{r}_{j}(t)\right] \\
& -\sum_{j=0}^{n} a_{i j} \gamma_{c} \varphi\left[\mathbf{v}_{i}(t)-\mathbf{v}_{j}(t)\right], \quad \forall i \in\{1, \ldots, n\},
\end{aligned}
$$

where $\mathbf{r}_{0}(t)$ and $\mathbf{v}_{0}$ are denoted as the position and velocity of the reference, respectively.

Theorem 3. Suppose that a group reference labeled as agent 0 is available to all agents of multiagent system (1). Using protocol (12) for multiagent system (1), then if the communication topology graph of (1) is undirected and connected, all agents can 
track the group reference and loose consensus can be achieved such that $\left\|\mathbf{v}_{i}(t)-\mathbf{v}_{0}(t)\right\|_{\infty} \leq \varepsilon_{v}$ and $\left\|\mathbf{r}_{i}(t)-\mathbf{r}_{0}(t)\right\|_{\infty} \leq \rho$ as $t \rightarrow \infty$, for all $i, j$, where $\rho$ is a certain bounded scalar.

Proof. Denote $\widetilde{\mathbf{r}}_{i}=\mathbf{r}_{i}-\mathbf{r}_{0}$ and $\widetilde{\mathbf{v}}_{i}=\mathbf{v}_{i}-\mathbf{v}_{0}$, for all $i \in\{1, \ldots, n\}$. Thus, protocol (12) can be written as

$$
\begin{aligned}
\dot{\widetilde{\mathbf{v}}}_{i}(t)= & -\sum_{j=0}^{n} a_{i j} \phi\left[\widetilde{\mathbf{r}}_{i}(t)-\widetilde{\mathbf{r}}_{j}(t)\right] \\
& -\sum_{j=0}^{n} a_{i j} \gamma_{c} \varphi\left[\widetilde{\mathbf{v}}_{i}(t)-\widetilde{\mathbf{v}}_{j}(t)\right], \quad \forall i \in\{1, \ldots, n\} .
\end{aligned}
$$

Note that (13) has the same expression form with (5), except for that the dummy variable $j$ in the summation function starts from 0 to $n$. Introduce a Lyapunov function as follows:

$$
\begin{aligned}
V= & \frac{1}{2} \sum_{i=1}^{n} \sum_{j=1}^{n} a_{i j} \mathbf{1}_{3}^{T} \psi\left(\widetilde{\mathbf{r}}_{i}-\widetilde{\mathbf{r}}_{j}\right) \\
& +\sum_{i=1}^{n} a_{i 0} \mathbf{1}_{3}^{T} \psi\left(\widetilde{\mathbf{r}}_{i}\right)+\frac{1}{2} \sum_{i=1}^{n} \widetilde{\mathbf{v}}_{i}^{T} \widetilde{\mathbf{v}}_{i} .
\end{aligned}
$$

Then the time derivative of $V$ along the trajectories of (13) is given by

$$
\begin{aligned}
& \dot{V}=\frac{1}{2} \sum_{i=1}^{n} \sum_{j=1}^{n} a_{i j}\left(\widetilde{\mathbf{v}}_{i}-\widetilde{\mathbf{v}}_{j}\right)^{T} \phi\left(\widetilde{\mathbf{r}}_{i}-\widetilde{\mathbf{r}}_{j}\right) \\
& +\sum_{i=1}^{n} a_{i 0} \widetilde{\mathbf{v}}_{i}^{T} \phi\left(\widetilde{\mathbf{r}}_{i}\right) \\
& -\sum_{i=1}^{n} \widetilde{\mathbf{v}}_{i}^{T}\left\{\sum_{j=1}^{n} a_{i j}\left[\phi\left(\widetilde{\mathbf{r}}_{i}-\widetilde{\mathbf{r}}_{j}\right)+\varphi\left(\widetilde{\mathbf{v}}_{i}-\widetilde{\mathbf{v}}_{j}\right)\right]\right. \\
& \left.+a_{i 0}\left[\phi\left(\widetilde{\mathbf{r}}_{i}\right)+\varphi\left(\widetilde{\mathbf{v}}_{i}\right)\right]\right\} \\
& =\sum_{i=1}^{n} \sum_{j=1}^{n} a_{i j} \widetilde{\mathbf{v}}_{i}^{T} \phi\left(\widetilde{\mathbf{r}}_{i}-\widetilde{\mathbf{r}}_{j}\right)+\sum_{i=1}^{n} a_{i 0} \widetilde{\mathbf{v}}_{i}^{T} \phi\left(\widetilde{\mathbf{r}}_{i}\right) \\
& -\sum_{i=1}^{n} \sum_{j=1}^{n} a_{i j} \widetilde{\mathbf{v}}_{i}^{T}\left[\phi\left(\widetilde{\mathbf{r}}_{i}-\widetilde{\mathbf{r}}_{j}\right)+\varphi\left(\widetilde{\mathbf{v}}_{i}-\widetilde{\mathbf{v}}_{j}\right)\right] \\
& -\sum_{i=1}^{n} a_{i 0} \widetilde{\mathbf{v}}_{i}^{T}\left[\phi\left(\widetilde{\mathbf{r}}_{i}\right)+\varphi\left(\widetilde{\mathbf{v}}_{i}\right)\right] \\
& =-\sum_{i=1}^{n} \sum_{j=1}^{n} a_{i j} \widetilde{\mathbf{v}}_{i}^{T} \varphi\left(\widetilde{\mathbf{v}}_{i}-\widetilde{\mathbf{v}}_{j}\right)-\sum_{i=1}^{n} a_{i 0} \widetilde{\mathbf{v}}_{i}^{T} \varphi\left(\widetilde{\mathbf{v}}_{i}\right) \\
& =-\frac{1}{2} \sum_{i=1}^{n} \sum_{j=1}^{n} a_{i j}\left(\widetilde{\mathbf{v}}_{i}-\widetilde{\mathbf{v}}_{j}\right)^{T} \varphi\left(\widetilde{\mathbf{v}}_{i}-\widetilde{\mathbf{v}}_{j}\right)
\end{aligned}
$$

$$
-\sum_{i=1}^{n} a_{i 0} \widetilde{\mathbf{v}}_{i}^{T} \varphi\left(\widetilde{\mathbf{v}}_{i}\right)
$$

$\leq 0$

where we have used derivative rules to obtain the first equality, have used Lemma 1 to obtain the third and last equality, and have used the fact that $\left(\mathbf{v}_{i}-\mathbf{v}_{j}\right)^{T} \varphi\left(\mathbf{v}_{i}-\mathbf{v}_{j}\right)$ and $\widetilde{\mathbf{v}}_{i}^{T} \varphi\left(\widetilde{\mathbf{v}}_{i}\right)$ are both non-negative for all $i$ and $j$ to get the final inequality.

Note that $\dot{V} \equiv 0$ means that $\left\|\widetilde{\mathbf{v}}_{i}(t)\right\|_{\infty} \leq \varepsilon_{v}$ and $\left\|\widetilde{\mathbf{v}}_{i}(t)-\widetilde{\mathbf{v}}_{j}(t)\right\|_{\infty} \leq \varepsilon_{v}$, for all $i, j$, and, by employing the similar analysis in Section 3.1, one can obtain that $\left\|\widetilde{\mathbf{r}}_{i}(t)\right\|_{\infty} \leq$ $\rho$. Since $\widetilde{\mathbf{r}}_{i}=\mathbf{r}_{i}-\mathbf{r}_{0}$ and $\widetilde{\mathbf{v}}_{i}=\mathbf{v}_{i}-\mathbf{v}_{0}$, it is clear that the proof is completed.

3.3. Offset Consensus with Partial Access to a Reference. In practice, it is more common that only a portion of agents can access to the reference, while others cannot. Generally in this case, the linear consensus problem often used the same form of protocol as what is used in the case where the group reference is available to all agents (see, e.g., $[3,5,6])$. However, in this work, protocol (12) cannot be direct borrowed to apply for loose consensus, and the reasons are as follows: partial access to the reference means that there exist some $i$ such that $a_{i 0}=0$, and hence $\sum_{i=1}^{n} a_{i 0} \widetilde{\mathbf{v}}_{i}^{T} \varphi\left(\widetilde{\mathbf{v}}_{i}\right)=0$ does not guarantee $\widetilde{\mathbf{v}}_{i}^{T} \varphi\left(\widetilde{\mathbf{v}}_{i}\right)=0$ for all $i$ any longer, and thus $\dot{V} \equiv 0$ will not lead to the results that $\left\|\widetilde{\mathbf{v}}_{i}(t)\right\|_{\infty} \leq \varepsilon_{v}$ for all $i$ (see the last equality of (15)). A worse outcome may occur, in which case, even though $\left\|\widetilde{\mathbf{v}}_{i}(t)-\widetilde{\mathbf{v}}_{j}(t)\right\|_{\infty} \leq \varepsilon_{v}$ holds for all $i$, there are still some $i$ for $\left\|\widetilde{\mathbf{v}}_{i}(t)\right\|_{\infty}>\varepsilon_{v}$ so that loose consensus is not achieved.

In order to better borrow the form of protocol (12), in this subsection, a set of $n$ distributed estimators are additionally designed as follows:

$$
\begin{aligned}
& \dot{\hat{\mathbf{x}}}_{i}=-\beta_{x} \operatorname{sgn}\left[\sum_{i=1}^{n} a_{i j}\left(\widehat{\mathbf{x}}_{i}-\widehat{\mathbf{x}}_{j}\right)+a_{i 0}\left(\widehat{\mathbf{x}}_{i}-\mathbf{r}_{0}\right)\right], \\
& \dot{\hat{\mathbf{y}}}_{i}=-\beta_{y} \operatorname{sgn}\left[\sum_{i=1}^{n} a_{i j}\left(\widehat{\mathbf{y}}_{i}-\widehat{\mathbf{y}}_{j}\right)+a_{i 0}\left(\widehat{\mathbf{y}}_{i}-\mathbf{v}_{0}\right)\right], \\
& \dot{\mathbf{\mathbf { z }}}_{i}=-\beta_{z} \operatorname{sgn}\left[\sum_{i=1}^{n} a_{i j}\left(\widehat{\mathbf{z}}_{i}-\widehat{\mathbf{z}}_{j}\right)+a_{i 0}\left(\widehat{\mathbf{z}}_{i}-\dot{\mathbf{v}}_{0}\right)\right],
\end{aligned}
$$

$\forall i \in\{1, \ldots, n\}$

where $\operatorname{sgn}(\cdot)$ denotes the signum function, defined component-wise. $\beta_{x}, \beta_{y}$, and $\beta_{z}$ are proper positive constants. $\widehat{\mathbf{x}}_{i}, \widehat{\mathbf{y}}_{i}$, and $\widehat{\mathbf{z}}_{i}$ are the $i$ th estimation calculated by agent $i$, and denote the estimation of position, velocity, and acceleration of the group reference, respectively.

It is worthwhile to note that estimators (16a), (16b), and (16c) are actually three independent linear first-order information consensus protocols. Also, note that what they deal with are not the dynamics or control forces but the information (the understandings of the reference state for 
each agent), so uncertainties do not exist and thus linear form is suitable for these estimators.

Theorem 4. Suppose that $\mathbf{v}_{0}, \dot{\mathbf{v}}_{0}$, and $\ddot{\mathbf{v}}_{0}$ are bounded as $\left\|\mathbf{v}_{0}\right\|_{\infty} \leq \rho_{x},\left\|\dot{\mathbf{v}}_{0}\right\|_{\infty} \leq \rho_{y}$, and $\left\|\ddot{\mathbf{v}}_{0}\right\|_{\infty} \leq \rho_{z}$, respectively. Using estimate algorithms (16a), (16b), and (16c) for multiagent system (1), consensus will be achieved in finite time such that $\widehat{\mathbf{x}}_{i} \rightarrow \widehat{\mathbf{x}}_{j} \rightarrow \mathbf{r}_{0}, \widehat{\mathbf{y}}_{i} \rightarrow \widehat{\mathbf{y}}_{j} \rightarrow \mathbf{v}_{0}$, and $\widehat{\mathbf{z}}_{i} \rightarrow \widehat{\mathbf{z}}_{j} \rightarrow \dot{\mathbf{v}}_{0}$, for all $i, j$, if and only if both the following conditions hold:

(i) the communication topology graph of (1) is undirected and connected, and there exists at least one $a_{i 0}>0$;

(ii) arguments satisfy that $\beta_{x}>\rho_{x}, \beta_{y}>\rho_{y}$ and $\beta_{z}>\rho_{z}$.

Proof (see [14] for more proving details). With $\widetilde{\mathbf{r}}_{i}=\widehat{\mathbf{x}}_{i}-\mathbf{r}_{0}$, we can rewrite $(16 a)$ as

$$
\dot{\mathbf{r}}_{i}=-\beta_{x} \operatorname{sgn}\left[\sum_{i=1}^{n} a_{i j}\left(\widetilde{\mathbf{r}}_{i}-\widetilde{\mathbf{r}}_{j}\right)+a_{i 0} \widetilde{\mathbf{r}}_{i}\right]-\dot{\mathbf{r}}_{0}(t) .
$$

Equation (17) can be written in matrix form as

$$
\dot{\tilde{\mathbf{r}}}=-\beta_{x} \operatorname{sgn}\left[\left(\mathbf{M} \otimes \mathbf{I}_{3}\right) \widetilde{\mathbf{r}}\right]-\mathbf{1}_{n} \otimes \dot{\mathbf{r}}_{0}(t),
$$

where $\widetilde{\mathbf{r}}$ is the column stack vector of $\widetilde{\mathbf{r}}_{i}, i=1, \ldots, n, \mathbf{M}=$ $\mathscr{L}+\operatorname{diag}\left(a_{10}, \ldots, a_{n 0}\right)$, and “ $\otimes$ " denotes the Kronecker product operator.

Note that the fixed undirected graph $\mathscr{G}$ is connected and at least one $a_{i 0}$ is positive; it follows that $\mathbf{M}$ is symmetric positive definite.

Consider the Lyapunov function candidate $V=(1 / 2) \widetilde{\mathbf{r}}^{T}\left(\mathbf{M} \otimes \mathbf{I}_{3}\right) \widetilde{\mathbf{r}}$. The derivative of $V$ is

$$
\begin{aligned}
\dot{V} & =\widetilde{\mathbf{r}}^{T}\left(\mathbf{M} \otimes \mathbf{I}_{3}\right)\left\{-\beta_{x} \operatorname{sgn}\left[\left(\mathbf{M} \otimes \mathbf{I}_{3}\right) \widetilde{\mathbf{r}}\right]-\mathbf{1}_{n} \otimes \dot{\mathbf{r}}_{0}(t)\right\} \\
& \leq-\beta_{x}\left\|\left(\mathbf{M} \otimes \mathbf{I}_{3}\right) \widetilde{\mathbf{r}}\right\|_{1}+\left|\dot{\mathbf{r}}_{0}(t)\right| \cdot\left\|\left(\mathbf{M} \otimes \mathbf{I}_{3}\right) \widetilde{\mathbf{r}}\right\|_{1} \\
& \leq-\left(\beta_{x}-\rho_{2}\right)\left\|\left(\mathbf{M} \otimes \mathbf{I}_{3}\right) \widetilde{\mathbf{r}}\right\|_{1} \\
& <0 .
\end{aligned}
$$

Here, we use the fact that $\mathbf{M}$ is symmetric positive definite, Holder's inequality, and $\beta_{x}>\rho_{2}$. It then follows from Lyapunov stability theory that $\|\widetilde{\mathbf{r}}\| \rightarrow \mathbf{0}$ as $t \rightarrow \infty$.

Note that $V=(1 / 2) \widetilde{\mathbf{r}}^{T}\left(\mathbf{M} \otimes \mathbf{I}_{3}\right) \widetilde{\mathbf{r}} \leq(1 / 2) \lambda_{\max }(\mathbf{M})\|\widetilde{\mathbf{r}}\|_{2}^{2}$; it then follows that the derivative of $V$ satisfies

$$
\begin{aligned}
\dot{V} & \leq-\left(\beta_{x}-\rho_{2}\right)\left\|\left(\mathbf{M} \otimes \mathbf{I}_{3}\right) \widetilde{\mathbf{r}}\right\|_{1} \\
& \leq-\left(\beta_{x}-\rho_{2}\right)\left\|\left(\mathbf{M} \otimes \mathbf{I}_{3}\right) \widetilde{\mathbf{r}}\right\|_{2} \\
& \leq-\left(\beta_{x}-\rho_{2}\right) \lambda_{\min }(\mathbf{M})\|\widetilde{\mathbf{r}}\|_{2} \\
& \leq-\left(\beta_{x}-\rho_{2}\right) \frac{\sqrt{2} \lambda_{\min }(\mathbf{M})}{\sqrt{\lambda_{\max }(\mathbf{M})}} \sqrt{V}
\end{aligned}
$$

After some manipulation, we get that

$$
2 \sqrt{V(t)} \leq 2 \sqrt{V(0)}-\left(\beta_{x}-\rho_{2}\right) \frac{\sqrt{2} \lambda_{\min }(\mathbf{M})}{\sqrt{\lambda_{\max }(\mathbf{M})}} t
$$

Therefore, there exists a finite time, which equals $\sqrt{\widetilde{\mathbf{r}}^{T}(0)\left(\mathbf{M} \otimes \mathbf{I}_{3}\right) \widetilde{\mathbf{r}}(0)} \sqrt{\lambda_{\text {max }}(\mathbf{M})} /\left(\beta_{x}-\rho_{2}\right) \lambda_{\text {min }}(\mathbf{M})$ actually, such that $V(t)=0$ holds for all $t$ after the finite time. Equations (16b) and (16c) can also be proved with no differences, and hence the proof is completed.

After the design of $n$ distributed estimators, all $n$ agents can have a consensus understanding of the state of the group reference after some finite time. In other words, after some finite time for such $n$ estimators to work, the group reference can be regarded as being available to all the $n$ agents by using their each estimation instead of the partial available group reference. And thus the remaining problem has been completely transformed to the case which is discussed in Section 3.2.

In this subsection, the configuration offset of each agent, which is denoted as $\delta_{i}$ for all $i$ (it can be time varying, and it should be self-known by agent $i$. Sometimes heterogeneous agents may be desired to form a given relative configuration. e.g., a sighted agent should be aware of its role of sensing, and hence an offset in front of the formation would be set as default when such an agent gets ready for formation motion), is also additionally considered. The loose consensus protocol for the $n$ agents, which have a partial access to a reference and have self-known offsets, is given by

$$
\begin{aligned}
\mathbf{u}_{i}(t)= & \widehat{\mathbf{z}}_{i}+\ddot{\delta}_{i}-\phi\left[\mathbf{r}_{i}(t)-\delta_{i}-\widehat{\mathbf{x}}_{i}\right]-\varphi\left[\mathbf{v}_{i}(t)-\dot{\delta}_{i}-\widehat{\mathbf{y}}_{i}\right] \\
& -\sum_{j=1}^{n} a_{i j} \phi\left[\mathbf{r}_{i}(t)-\delta_{i}-\mathbf{r}_{j}(t)+\delta_{j}\right] \\
& -\sum_{j=1}^{n} a_{i j} \gamma_{c} \varphi\left[\mathbf{v}_{i}(t)-\dot{\delta}_{i}-\mathbf{v}_{j}(t)+\dot{\delta}_{j}\right],
\end{aligned}
$$$$
\forall i \in\{1, \ldots, n\}
$$

Theorem 5. After using (16a), (16b), and (16c) for multiagent system (1) for an enough time $t<T_{0}$, employing protocol (22), if both the conditions in Theorem 4 hold, then system (1) will achieve loose consensus such that $\left\|\mathbf{v}_{i}(t)-\dot{\delta}_{i}-\mathbf{v}_{0}(t)\right\|_{\infty} \leq \varepsilon_{v}$ and $\left\|\mathbf{r}_{i}(t)-\delta_{i}-\mathbf{r}_{0}(t)\right\|_{\infty} \leq \rho$ as $t \rightarrow \infty$, for all $i, j$, where $\rho$ is a certain bounded scalar.

Proof. If both the conditions in Theorem 4 hold, then it follows from Theorem 4 that there exists $T_{0}>0$ such that $\widehat{\mathbf{x}}_{i} \rightarrow \widehat{\mathbf{x}}_{j} \rightarrow \mathbf{r}_{0}, \widehat{\mathbf{y}}_{i} \rightarrow \widehat{\mathbf{y}}_{j} \rightarrow \mathbf{v}_{0}$, and $\widehat{\mathbf{z}}_{i} \rightarrow \widehat{\mathbf{z}}_{j} \rightarrow \dot{\mathbf{v}}_{0}$, for all $t>T_{0}$.

Thus, for all $t>T_{0}$, we can use $\mathbf{r}_{0}, \mathbf{v}_{0}$, and $\dot{\mathbf{v}}_{0}$ to take the place of $\widehat{\mathbf{x}}_{i}, \widehat{\mathbf{y}}_{i}$, and $\widehat{\mathbf{z}}_{i}$, respectively, in (22). And by denoting $\widetilde{\mathbf{r}}_{i}=\mathbf{r}_{i}-\delta_{i}$ and $\widetilde{\mathbf{v}}_{i}=\mathbf{v}_{i}-\dot{\delta}_{i},(22)$ can be written as follows:

$$
\begin{gathered}
\dot{\widetilde{\mathbf{v}}}_{i}(t)=\dot{\mathbf{v}}_{0}-\phi\left[\widetilde{\mathbf{r}}_{i}(t)-\mathbf{r}_{0}\right]-\varphi\left[\widetilde{\mathbf{v}}_{i}(t)-\mathbf{v}_{0}\right] \\
-\sum_{j=1}^{n} a_{i j} \phi\left[\widetilde{\mathbf{r}}_{i}(t)-\widetilde{\mathbf{r}}_{j}(t)\right]
\end{gathered}
$$




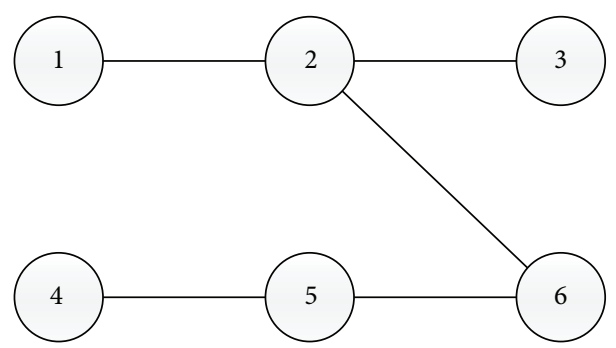

(a)

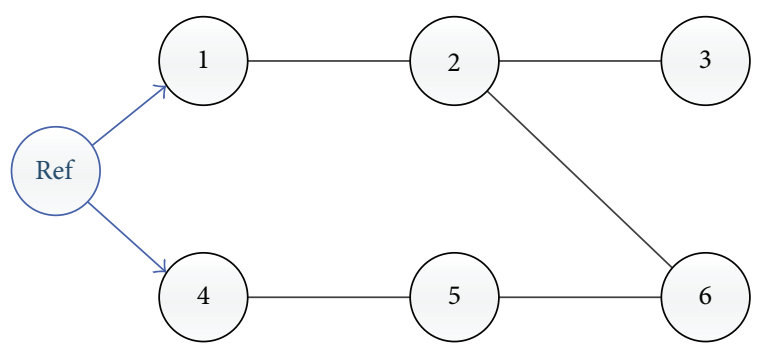

(b)

FIGURE 1: Communication links of a multiagent system.

$$
\begin{gathered}
-\sum_{j=1}^{n} a_{i j} \gamma_{c} \varphi\left[\widetilde{\mathbf{v}}_{i}(t)-\widetilde{\mathbf{v}}_{j}(t)\right] \\
=\dot{\tilde{\mathbf{v}}}_{0}(t)-\sum_{j=0}^{n} a_{i j} \phi\left[\widetilde{\mathbf{r}}_{i}(t)-\widetilde{\mathbf{r}}_{j}(t)\right] \\
-\sum_{j=0}^{n} a_{i j} \gamma_{c} \varphi\left[\widetilde{\mathbf{v}}_{i}(t)-\widetilde{\mathbf{v}}_{j}(t)\right] .
\end{gathered}
$$

Note that, if one let all $a_{i 0}$ in (12) equal 1 , then (12) can be totally equivalent to (23). Therefore, the same process of analysis in Section 3.2 can be borrowed to complete the proof, and hence detailed process can be omitted here. It can be followed from the conclusion of Theorem 3 that $\left\|\widetilde{\mathbf{v}}_{i}(t)-\mathbf{v}_{0}(t)\right\|_{\infty} \leq \varepsilon_{v}$ and $\left\|\widetilde{\mathbf{r}}_{i}(t)-\mathbf{r}_{0}(t)\right\|_{\infty} \leq \rho$. Note that $\widetilde{\mathbf{r}}_{i}=$ $\mathbf{r}_{i}-\delta_{i}$ and $\widetilde{\mathbf{v}}_{i}=\mathbf{v}_{i}-\dot{\delta}_{i}$, and it is clear that $\left\|\mathbf{v}_{i}(t)-\dot{\delta}_{i}-\mathbf{v}_{0}(t)\right\|_{\infty} \leq$ $\varepsilon_{v}$ and $\left\|\mathbf{r}_{i}(t)-\delta_{i}-\mathbf{r}_{0}(t)\right\|_{\infty} \leq \rho$, which means that the offset of agents will achieve loose consensus as $t \rightarrow \infty$.

\section{Numerical Simulations}

In the simulations, we will show that the proposed protocol (5) and (22) can lead to consensus firstly. Then by comparing protocols (5) with (2), we will show that, in the existence of measurement uncertainties, the proposed protocols in this paper are of benefit in saving energy.

A multiagent system which contains 6 agents is taken as an example, and they are considered in 1 dimension for simplicity, although the analyses throughout the paper are discussed in any dimensions of order $m$. Figure 1(a) shows the internal communication links in the system, while Figure 1(b) additionally contains the communication links of the external reference. The weights of each edge are all set to 0.8 . Initial conditions of the 6 agents are all set randomly, which are $\mathbf{r}\left(t_{0}\right)=[-4,5,-7,4,-3,3]^{T} \mathrm{~m}$ and $\mathbf{v}\left(t_{0}\right)=[1.6,2.2,-1.5,3,-2.8,-0.8]^{T} \mathrm{~m} / \mathrm{s}$ as an instantiation in particular. The arguments in function (4) are set as $\varepsilon_{r}=0.05$ and $\varepsilon_{v}=0.02$, which means that measurement uncertainties of positions are within $0.05 \mathrm{~m}$ and those of velocities are within $0.02 \mathrm{~m} / \mathrm{s}$. The damping scalar $\gamma_{c}$ is set to 2.5. The group reference is set as $r_{0}(t)=2 \sin (0.2 t)$, and the offsets of the $i$ th agent from the reference are $i \mathrm{~m}$, respectively, which are constant.

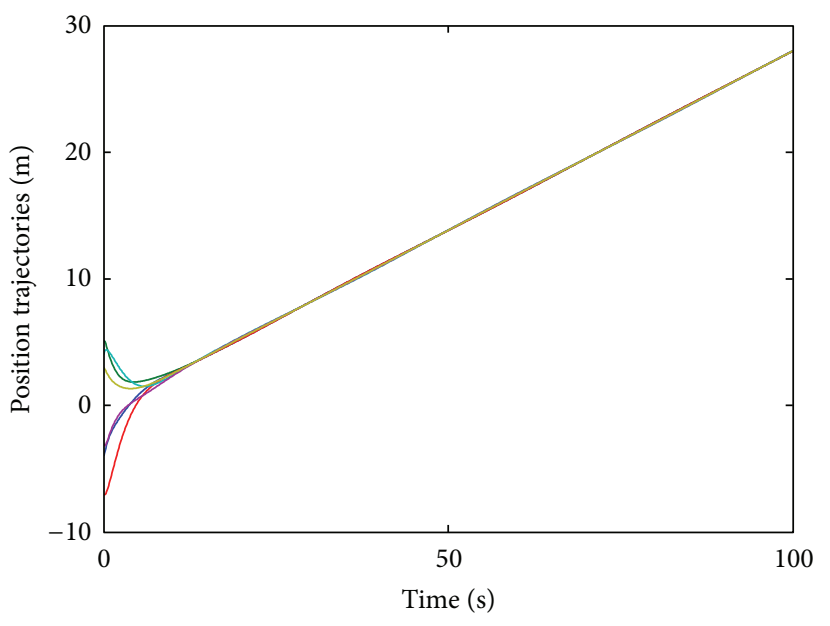

Figure 2: Position trajectories of all agents with (5).

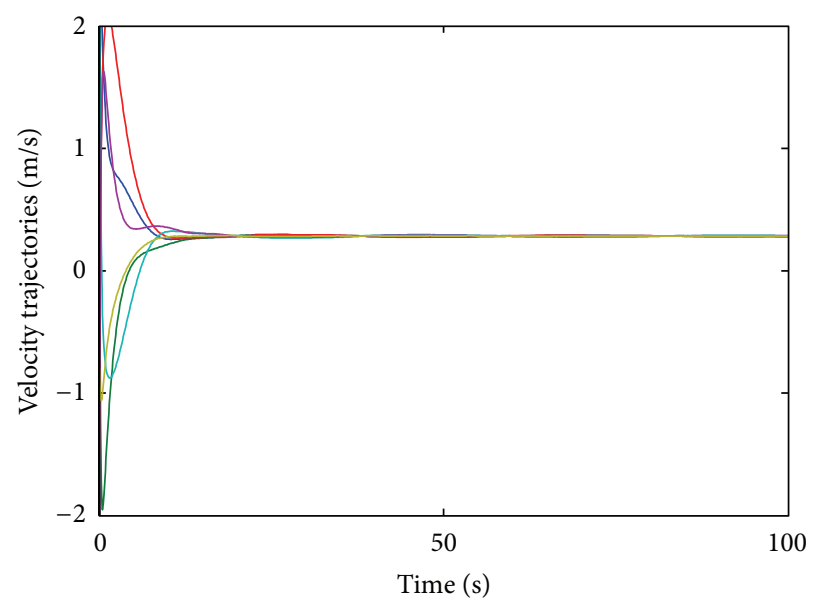

FIGURE 3: Velocity trajectories of all agents with (5).

When a group reference does not exist, employing protocol (5) for the system, we get simulation results shown in Figures 2 and 3. It is clear that the 6 agents gather and then keep moving together with similar velocities around their average velocity. Since their positions and velocities are very similar but not exact coincident, we know that loose consensus is achieved. The trajectories results by applying 
linear protocol (2) are similar to Figures 2 and 3 with little difference actually, so they are omitted here.

When the reference exists, after using (16a), (16b), and (16c) to estimate the reference for a period of time, we employ protocol (22) for the system, then we get simulation results shown in Figures 4 and 5. It can be seen that, in the existence of the reference, the trajectories of agents are different from what have been shown in Figures 2 and 3. The position trajectories change like sinusoidal functions with amplitudes of about $4 \mathrm{~m}$, and velocity trajectories also change like sinusoidal functions with amplitudes of about $0.8 \mathrm{~m} / \mathrm{s}$. Both the position trajectories and velocity trajectories are in accordance with the given reference. It can be known that agents track the reference successfully. And the difference among each position trajectory is their different offsets; the phenomenon shows that the self-known configuration offsets do have an effect. Loose consensus is achieved.

Then we compare linear protocol (2) and non-linear protocol (5) in the area of energy saving. We introduce a quantity that indicates the sum of the energy (e.g., fuels) of all the agents consuming to generate forces over time, defined as $E(t)=\int_{0}^{t} \sum_{i=1}^{n}\left|u_{i}\right| d x$. Comparison results are shown in Figure 6. For clear statement, the simulation time $(0 \sim 100 \mathrm{~s})$ can be distinguished into two periods: in the first period, which can be called formation forming in this paper, agents try to achieve consensus from distinct initial states till the consensus achieved; and in the second one, which can be called formation keeping in this paper, agents maintain their similar states. It can be seen that, in the period of formation forming, there is nearly no difference between the two protocols. And as soon as the multiagent system gets into the period of formation keeping, protocol (5) performs better to save energy, which costs much less energy than protocol (2) does. The reason is obvious in that, since the measuring errors exist, linear protocol (2) finds it hard to make agents agree on the very exact average and thus cost energy continuously. As a comparison, nonlinear protocol (5) allows agents to achieve a loose agreement, and hence it avoids the unnecessary cost of energy. It is also worthwhile to note that protocol (5) is more effective in the keeping period but less effective in the forming period. This is because, in the forming period, compared to measurement uncertainties, the state differences among agents are large enough to mask the effect of the loose control. In this sense, the protocol proposed in this work will have no advantage compared to the linear one in the case where relative configuration of formation is desired to be time varying.

\section{Conclusions}

In this paper, in order to avoid a potential waste of energy in the case where there exist measurement uncertainties, a nonlinear protocol is proposed with a connected undirected communication topology. The protocol is then extended to both the cases where agents can have full or partial access to a group reference. Especially in the case of partial access, a set of distributed estimators is utilized to help all agents agree on the understandings of the reference, even though

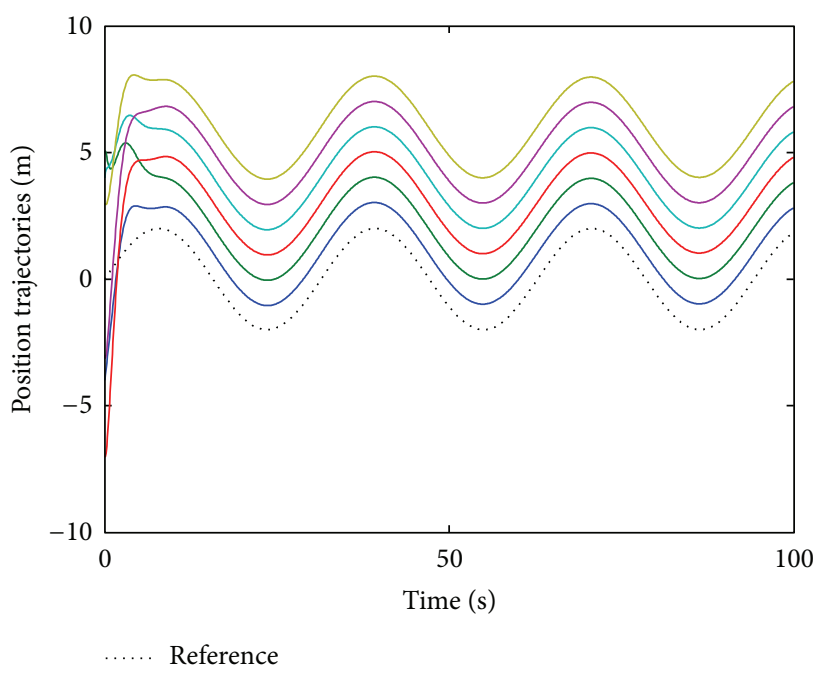

FIgURE 4: Position trajectories of all agents with (22).

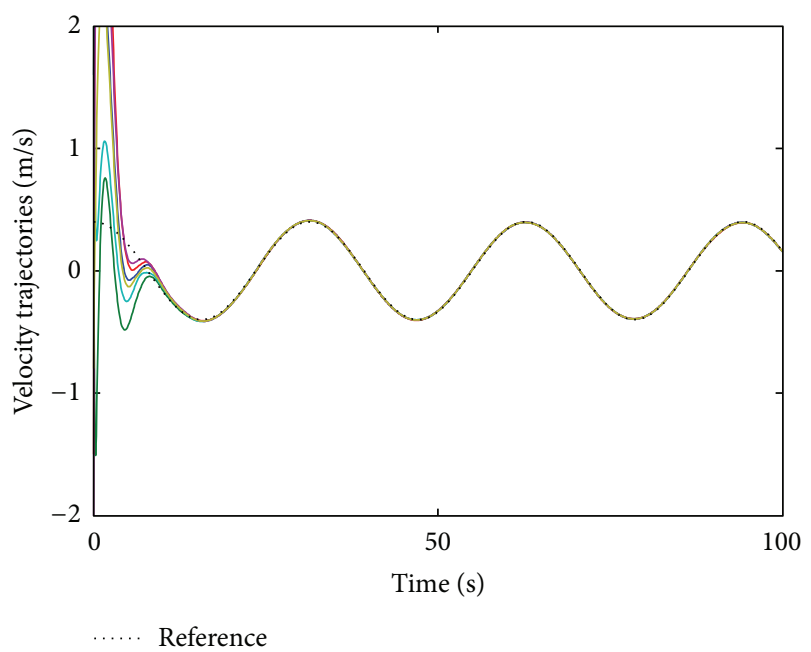

FIgURE 5: Velocity trajectories of all agents with (22).

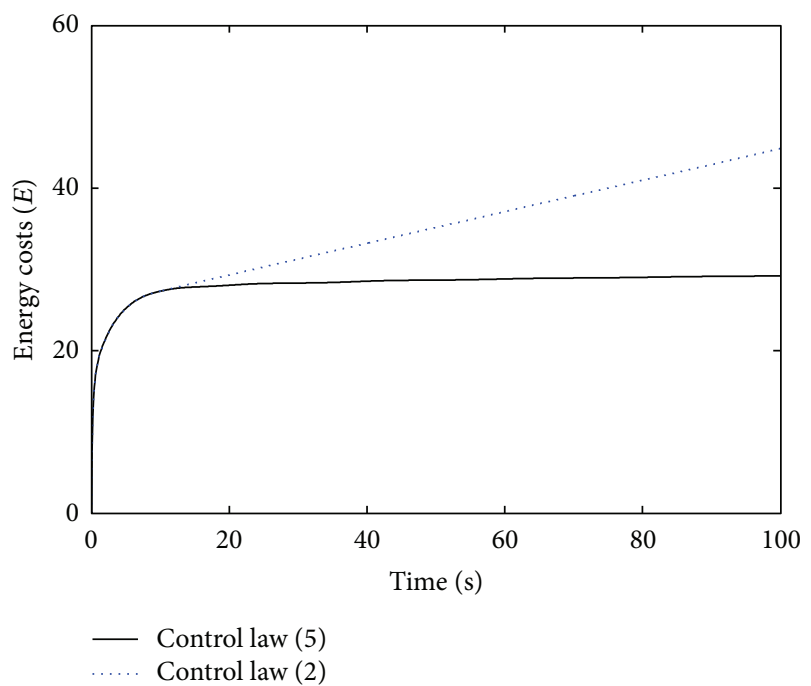

FIGURE 6: Comparison of energy costs. 
some agents cannot access to the reference directly. An additional condition where self-known configuration offsets are desired is also considered. Theoretical analyses of stability are given for the proposed protocols. Finally, simulations are performed by MATLAB, and results show that these protocols can lead agents to loose consensus and work effectively with less energy cost to keep formation, while there is little enhancement by using such protocols when agents are trying to form the formation.

\section{References}

[1] A. Jadbabaie, J. Lin, and A. S. Morse, "Coordination of groups of mobile autonomous agents using nearest neighbor rules," IEEE Transactions on Automatic Control, vol. 48, no. 6, pp. 988-1001, 2003.

[2] W. Ren, R. W. Beard, and E. M. Atkins, "Information consensus in multivehicle cooperative control," IEEE Control Systems Magazine, vol. 27, no. 2, pp. 71-82, 2007.

[3] W. Ren, "On consensus algorithms for double-integrator dynamics," IEEE Transactions on Automatic Control, vol. 53, no. 6, pp. 1503-1509, 2008.

[4] R. O. Saber, "Flocking for multi-agent dynamic systems: algorithms and theory," IEEE Transactions on Automatic Control, vol. 51, no. 3, pp. 401-420, 2006.

[5] Z. Meng, Z. Lin, and W. Ren, "Leader-follower swarm tracking for networked Lagrange systems," Systems and Control Letters, vol. 61, no. 1, pp. 117-126, 2012.

[6] W. Ren, "Formation keeping and attitude alignment for multiple spacecraft through local interactions," Journal of Guidance, Control, and Dynamics, vol. 30, no. 2, pp. 633-638, 2007.

[7] Y. Cao, D. Stuart, W. Ren, and Z. Meng, "Distributed containment control for multiple autonomous vehicles with doubleintegrator dynamics: algorithms and experiments," IEEE Transactions on Control Systems Technology, vol. 19, no. 4, pp. 929938, 2011.

[8] R. O. Saber and R. M. Murray, "Consensus protocols for networks of dynamic agents," in Proceedings of the American Control Conference, pp. 951-956, June 2003.

[9] D. Bauso, L. Giarré, and R. Pesenti, "Non-linear protocols for optimal distributed consensus in networks of dynamic agents," Systems and Control Letters, vol. 55, no. 11, pp. 918-928, 2006.

[10] F. Xiao, L. Wang, and Y. Jia, "Fast information sharing in networks of autonomous agents," in Proceedings of the American Control Conference (ACC '08), pp. 4388-4393, Seattle, Wash, USA, June 2008.

[11] H. Pan, X. Nian, and L. Guo, "Second-order consensus in multiagent systems via nonlinear protocol," Mathematical Problems in Engineering, vol. 2013, Article ID 978251, 7 pages, 2013.

[12] G. Purcell, D. Kuang, S. Lichten, S.-C. Wu, and L. Young, "Autonomous formation flyer (AFF) sensor technology development," TMO Progress Report, 1998.

[13] W. Ren, R. W. Beard, and E. M. Atkins, "Information consensus in multivehicle cooperative control: collective group behavior through local interaction," IEEE Control Systems Magazine, vol. 27, no. 2, pp. 71-82, 2007.

[14] Y. Cao, W. Ren, and Z. Meng, "Decentralized finite-time sliding mode estimators and their applications in decentralized finitetime formation tracking," Systems and Control Letters, vol. 59, no. 9, pp. 522-529, 2010. 


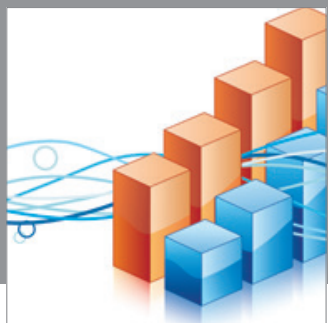

Advances in

Operations Research

mansans

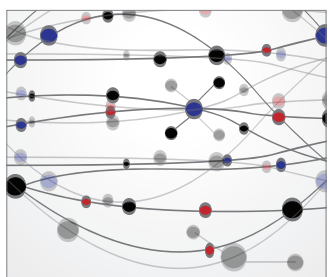

The Scientific World Journal
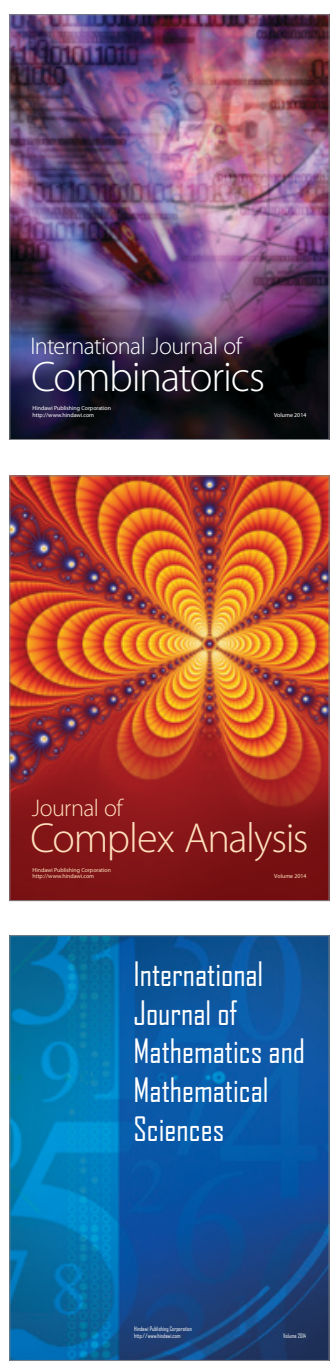
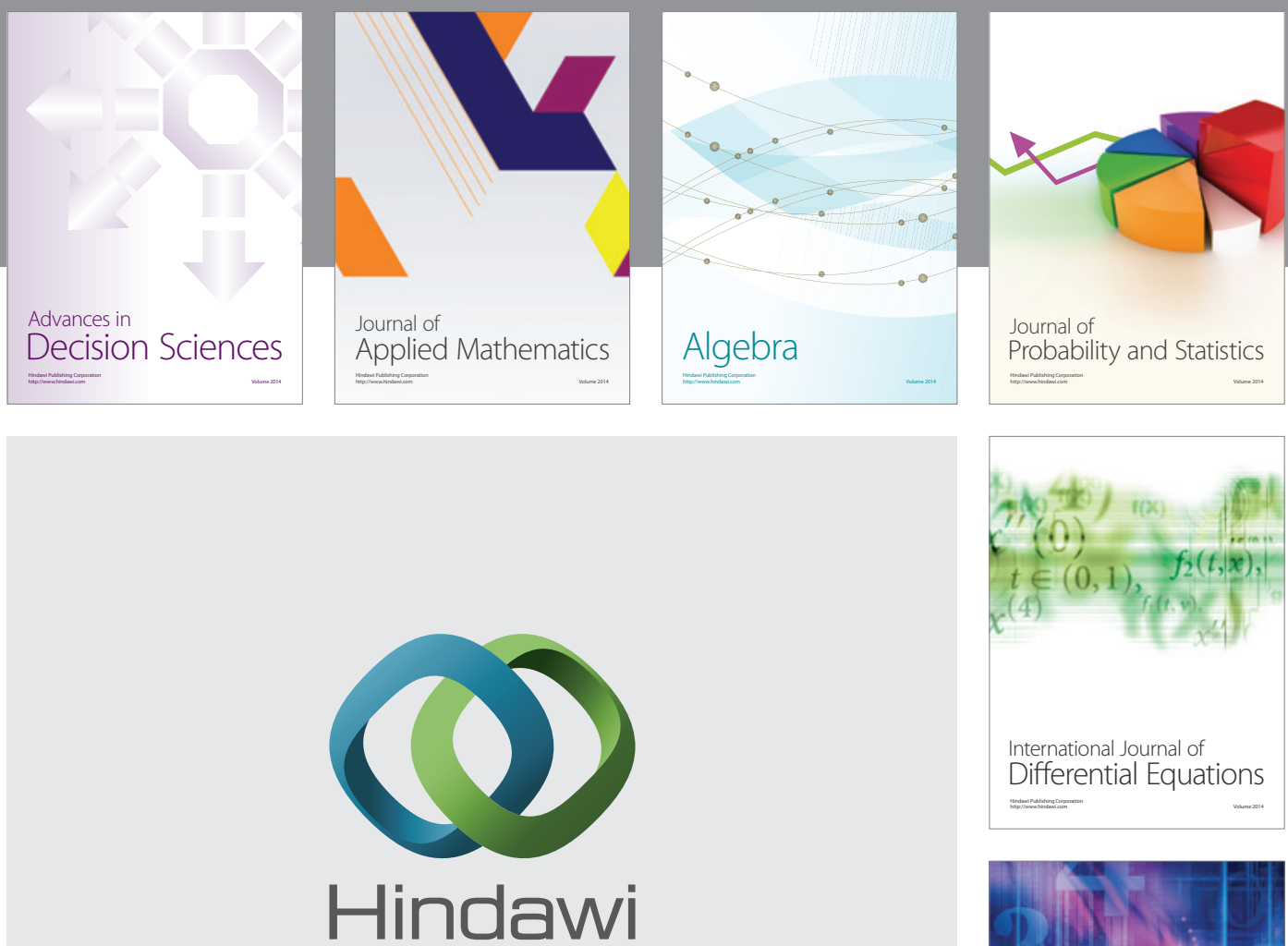

Submit your manuscripts at http://www.hindawi.com
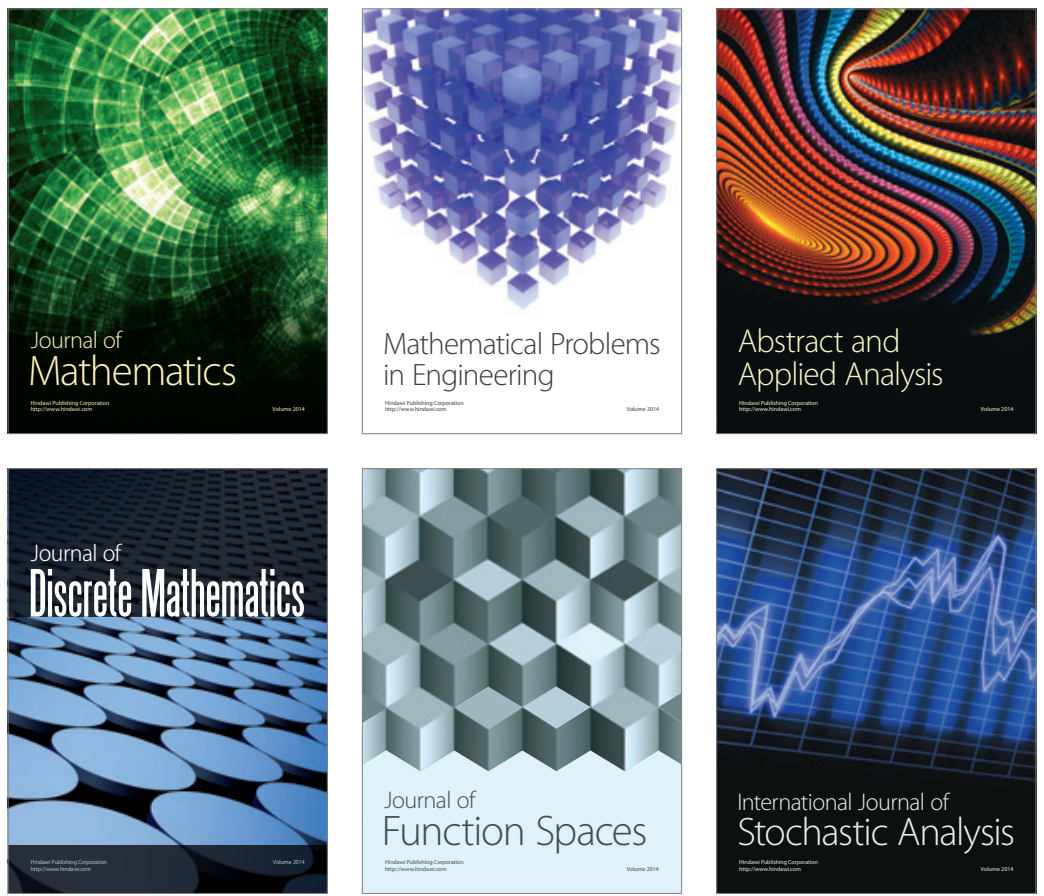

Journal of

Function Spaces

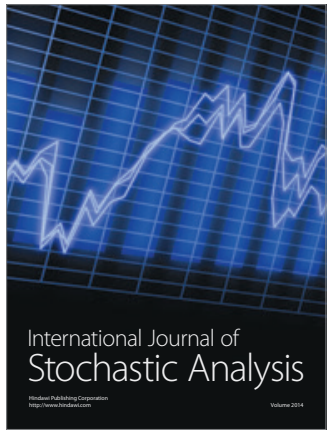

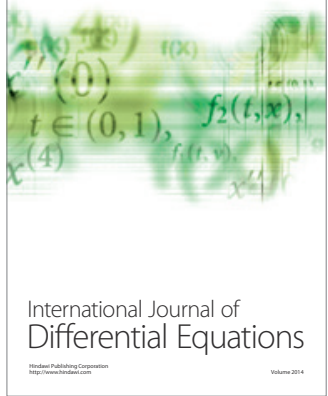
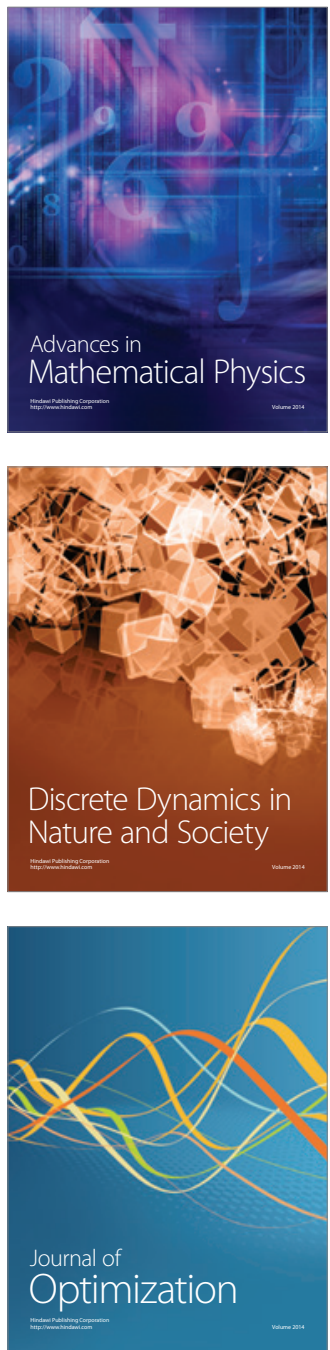